\title{
Emergência de Classes Seqüenciais Após Treino com Pareamento Consistente ${ }^{1}$
}

\author{
Mylena Pinto Lima e Grauben Assis ${ }^{2}$ \\ Universidade Federal do Pará
}

\begin{abstract}
RESUMO - Relações de ordem podem ser documentadas por meio de testes comportamentais através das propriedades de assimetria, transitividade e conectividade. A emergência de classes seqüenciais pode ser estabelecidas de diferentes maneiras, inclusive a partir do matching to sample com pareamento consistente de estímulos e sem conseqüências imediatas. O presente estudo buscou verificar o efeito do treino com pareamento consistente entre estímulos visuais sobre desempenhos emergentes. Cinco universitários de ambos os sexos foram submetidos ao treino das relações condicionais $A B, A C$ e $A D$. A tarefa dos participantes era responder ordinalmente a dígitos e formas geométricas abstratas. Em seguida, os participantes foram expostos a testes para ordenação de três sequiências diferentes com cinco estímulos. Três participantes alcançaram o critério de acerto e apresentaram um responder consistente nos testes. Os resultados indicaram que o treino foi efetivo no estabelecimento de relações de ordem entre estímulos e replicam dados da literatura no estabelecimento de desempenho seqüencial após treino com matching to sample.
\end{abstract}

Palavras chave: pareamento consistente; equivalência de estímulos; classes seqüenciais, humanos.

\section{Sequential Classes Emergence After Consistent Matching Training}

\begin{abstract}
Order relations can be documented by behavioral tests that evaluate whether such relations posses the properties of asymmetry, transitivity and connectedness. We used matching to sample with prerequisites for the emergence of sequential class. In the present study, we assess whether sequential classes were also produced after a trial with consistent matching, without immediate differential consequences. Five undergraduate students of both sexes were submitted to the training of conditional relations $\mathrm{AB}, \mathrm{AC}$ and $\mathrm{AD}$. The participants task were to choose abstract geometric figure from digits. After teaching the conditional relations, the participants were exposed to tests of ordering the stimuli in three sequences with five different stimuli, tests for ordering pairs of the stimuli that occupied adjacent and not adjacent positions in the sequence of five stimuli, and tests of multiple substitutions of stimuli, without additional training. Three participants reached the learning criterion in the training of the conditional relations and obtained high number of successes in the sequential tests. The consistency training without immediate differential consequences was effective to establish order relations among stimuli, without explicit training with normal adult participants. These results replicate data reported the literature on the establishment of sequential performance after matching to sample training.
\end{abstract}

Key words: consistency training; stimulus equivalence; sequential classes; humans.

Neste trabalho, vamos considerar que uma interpretação analítico-comportamental do comportamento deva estar baseada na análise funcional de desempenhos produtivos, aqueles desempenhos que não foram diretamente ensinados e que podem ser investigados através da análise da formação de classes de estímulos. Uma classe de estímulos pode ser verificada a partir do estabelecimento de relações entre estímulos singulares, como objetos, eventos ou qualidades diversas (cf. Sidman, 1986; 1994) e tem sido produzida através de um procedimento de matching to sample. Este procedimento consiste na apresentação de um estímulo condicional

1 Este trabalho é parte da Dissertação de Mestrado defendida pelo primeiro autor, sob orientação do segundo, no Programa de Pós-Graduação em Teoria e Pesquisa do Comportamento/UFPA. e contou com o apoio financeiro da CAPES. Os autores agradecem as sugestões e críticas dos professores Dr. Jair Lopes Junior (UNESP-Baurú) e Dr. Marcelo Galvão Baptista (UFPA.). O software de controle e análise dados comportamentais foi desenvolvido pela programadora Ane Margarete Monte Verde Silva, a quem os autores agradecem. (modelo) seguido de dois ou mais estímulos discriminativos (comparações) e na apresentação de conseqüências diferenciais para a escolha do estímulo de comparação positivo (cf. Sidman \& Tailby, 1982).

O estabelecimento de relações arbitrárias entre estímulo, na ausência da mediação por resposta comum e que ocasiona a formação de classes, é produzido através do procedimento de arbitrary matching to sample. Neste procedimento, além do estabelecimento de relações condicionais entre estímulos fisicamente diferentes, através do ensino direto, novas relações condicionais podem emergir, sem que tenham sido ensinadas diretamente, isto é ocorre a formação de classes de estímulos equivalentes. Diferentes arranjos de treino podem ser organizados no pareamento arbitrário com o modelo para o estabelecimento de classes de equivalência. A investigação dos efeitos dessas variações estruturais relacionam-se com uma diversidade de tipos de controle entre estímulos (cf. Fields \& Verhave, 1987; Carrigan \& Sidman, 1992; Saunders $\&$ Green, 1999). Dentre os trabalhos que buscaram esclarecer as condições de instalação e manutenção de relações condicionais e relações de equivalência situam-se os estudos 
que investigam o estabelecimento de relações condicionais, na ausência de consequiências diferenciais imediatas. Estes usam como estratégia alternativa, o pareamento consistente de estímulos modelo-comparação positiva (Sc), onde o estímulo modelo passa a ser emparelhado com um estímulo de comparação que permanece presente em todas as tentativas e os demais variando sistematicamente, como forma de garantir (ou tornar mais provável) a escolha do estímulo de comparação programado como positivo (cf. Harrison \& Green, 1990; Williams, Saunders, Saunders \& Spradlin, 1995; Baptista \& Assis, 1995; Assis, Baptista, Damin \& Álvares, 1997; Barros, Baptista \& Assis, 1998; Damin; Assis \& Baptista; 1998; Saunders, Drake \& Spradlin, 1999; Assis, Baptista, Kato \& Alves, 2000; Álvares, Assis, Sampaio \& Esteves, 2001).

Recentemente, uma nova proposição para análise de relações entre estímulos tem sido investigada. Busca-se analisar relações de ordem estabelecidas entre estímulos que ocorrem em uma seqüência de respostas e entre seqüências ensinadas independentemente, sugerindo a emergência de novas seqüências, não treinadas. (Green, Stromer \& Mackay, 1993; Maydak, Stromer, Mackay \& Stoddard, 1995; Holcomb, Stromer \& Mackay, 1997).

A proposta para análise do desempenho seqüencial produtivo sugerida por Green e cols. (1993), pode ser descrita como uma extensão do paradigma de equivalência de estímulos, com ênfase na análise de relações ordinais estabelecidas entre estímulos dentro de uma única seqüência ou entre sequiências independentes. A avaliação do desempenho seqüencial produtivo implica na documentação das propriedades de uma relação de ordem, quais sejam: irreflexividade, assimetria, transitividade e conectividade. Estas propriedades, assim como as propriedades de uma relação de equivalência (i. e: reflexividade, simetria e transitividade) são derivadas da matemática e caracterizam-se por favorecer a mensuração de desempenhos comportamentais específicos.

Duas das principais implicações desta proposta de análise do desempenho seqüencial relaciona-se com os fenômenos de encadeamento de respostas e controle condicional. Na interpretação de Green e cols. (1993), desempenhos seqüenciais baseados em uma relação de ordem, implicam na formação de classes de estímulos funcionalmente equivalentes; dessa forma, não podem estar baseadas nas mesmas relações encontradas em uma cadeia comportamental, onde as respostas sucessivas são integradas através de estímulos (elos) exercendo uma dupla função: discriminativa e reforçadora condicionada (Skinner, 1953); ou em uma relação condicional discreta, onde discriminações simples estão sob controle de um estímulo condicional (Sidman, 1986).

Um evento que envolva desempenhos seqüenciais produtivos deve também envolver a formação de classes de estímulos funcionalmente equivalentes. Mas, diferenciando-se da proposta de análise de classes verbais com ênfase na emergência de "significados" como produto da formação de classes de estímulos equivalentes (semântica), a análise de desempenhos seqüenciais produtivos sugere um campo de investigação sobre a organização de frases e sentenças (sintaxe). A ênfase deste tipo de análise requer inicialmente, a compreensão da natureza das relações de ordem que podem ser estabelecidas entre estímulo membros de uma seqüência e entre estímulos membros de seqüências diferentes. Esta não é uma questão recente na analise experimental do comportamento e diversos autores já forneceram contribuições valiosas, como Catania (1980) quando refere-se à produtividade do responder discriminado: "If stimulus properties control behavior sequentially, novel response sequences and generated directly by novel combinations of stimulus properties" (p.181) ${ }^{2}$.

Skinner (1957) na análise da gramática e sintaxe como processos autoclíticos, afirma:

The ordering and grouping of responses also have several functions. In the first place, speech sounds are ordered in the patterning of responses. Apart from the spectra of single speech sounds, the only dimension of verbal behavior is temporal, and order is therefore an important property (p. 333). ${ }^{3}$

Atualmente, considera-se que a análise de relações de ordem estabelecidas entre estímulos deva compreender a análise da formação de classes seqüenciais. Por isso, classes seqüências são a principal unidade de análise no estudo das relações entre estímulos dentro de seqüências (Lazar, 1977, Sigurdardottir, Green, \& Saunders, 1990; Stromer \& Mackay, 1990; 1992; 1993; Green, Stromer \& Mackay, 1993). Green et al. (1993) descrevem o estudo da formação de classes sequiências como um tipo de análise de desempenhos produtivos com duas características principais: ser uma proposta análoga conceitual e metodologicamente ao paradigma de equivalência de estímulos e ter implicações tanto teóricas como práticas para estudos relacionados com o comportamento humano complexo.

Na proposta de Green e cols. (1993), a questão que se coloca é se os sujeitos ensinados a responder a estímulos dentro de uma seqüência poderiam produzir seqüências não treinadas diretamente ou relações condicionais envolvendo estímulos específicos.

Para responder a esta questão empiricamente, utiliza-se testes comportamentais para as propriedades de uma relação ordinal tomadas a partir da sua definição na matemática, bem como procedimentos de avaliação da natureza das relações estímulo-estímulo dentro da seqüência e entre seqüências treinadas independentemente uma da outra. A definição matemática de uma relação ordinal foi tomada como guia para uma análise descritiva e organizada de relações estímuloestímulo produzidos por um treino seqüencial. São usados códigos alfanuméricos para representar estímulos e o símbolo => para indicar uma relação de ordem, tal como "vem antes". Por exemplo, $\mathrm{A} 1=>\mathrm{A} 2=>\mathrm{A} 3=>\mathrm{A} 4=>\mathrm{A} 5$ representa cinco estímulos diferentes respondidos numa dada ordem.

A investigação está baseada em testes comportamentais que avaliam se relações entre estímulos dentro da seqüência e entre sequiências treinadas independentemente possuem as propriedades de relações ordinais quais sejam: irreflexivi-

2 Se propriedades de estímulos controlam o comportamento sequencialmente, novas seqüências de resposta são geradas diretamente por novas combinações de propriedades de estímulos (p.181).

3 A ordenação e agrupamento de respostas também tem várias funções. Em primeiro lugar, os sons da fala são ordenados na modelagem de respostas. Além do espectro dos simples sons da fala, a única dimensão do comportamento verbal é temporal e, por isso, a ordem é uma propriedade importante (p.333). 
dade, assimetria, transitividade e conectividade. De acordo com a matemática, irreflexividade pressupõe que uma relação ordinal não é explicitamente reflexiva; não é verdade, por exemplo, que A1=>A1. A propriedade da assimetria pressupõe que uma relação ordinal deve ser unidirecional, neste caso se $\mathrm{A} 2=>\mathrm{A} 3$, então $\mathrm{A} 3=>\mathrm{A} 2$ não pode ser considerado correto. A transitividade pode ser considerada numa relação ordinal se, por exemplo, A2=>A3 e A3=>A4, então $\mathrm{A} 2=>\mathrm{A} 4$. Note-se que apenas pares de estímulos que não são adjacentes dentro de séries treinadas podem ser a base para a inferência desta propriedade. E, finalmente, a propriedade de conectividade pressupõe que uma relação existe se é correlata para todos os pares de estímulos dentro de um dado campo. Ressalta-se que relações deste tipo são necessárias mas sua identificação não é suficiente para o arranjo do que ocorre dentro de uma sequiência. Na propriedade conectividade, se $\mathrm{A} 1=>\mathrm{A} 2=>\mathrm{A} 3$, então $\mathrm{A} 1=>\mathrm{A} 2, \mathrm{~A} 1=>\mathrm{A} 3$ e $\mathrm{A} 2=>\mathrm{A} 3$. Como se vê, esta propriedade somente é inferida se todos os pares são possíveis, pares não adjacentes dos quais a transitividade é inferida e pares adjacentes. Desta forma, esta proposta provê uma estratégia para o exame da interação entre equivalência e relações ordinais quando resultam na expansão de repertórios comportamentais, que ocorrem em condições apropriadas. Uma relação que apresente as propriedades se assimetria e transitividade também apresentará a propriedade de irreflexividade.

Um dado importante proveniente dos resultados obtidos em estudos com o paradigma da equivalência é que a mediação verbal parece não ser necessária em alguns desempenhos de matching to sample e que o mesmo processo comportamental parece estar envolvido tanto no desenvolvimento de equivalência de estímulos como no comportamento verbal. Assim, uma interpretação analítico comportamental da semântica poderia sugerir que objetos, sons e palavras impressas relacionadas num mesmo evento tornam-se estímulos equivalentes a partir de sua participação no que se conhece como overlapping conditional relation (Adams, Fields \& Verhave, 1993). Nesta interpretação, as relações entre estímulos estabelecidas dentro de sequiências estaria provendo a base para o desenvolvimento da ordenação de palavras; quando por exemplo, palavras ocorressem numa mesma posição ordinal em diferentes sequiências, tornarse-iam mutuamente intercambiáveis ou funcionalmente equivalentes favorecendo a produção de novas sequiências de palavras. Em um exemplo do cotidiano um indivíduo a quem é ensinado algo como as sentenças "A casa é grande", "Uma bola azul" e "Esta blusa suja", seria capaz de produzir sentenças novas, gramaticalmente corretas, consistindo de várias recombinações de palavras dentro das seqüências ensinadas, por exemplo "Esta casa é azul", "A bola suja" ou "Uma blusa grande", sem ensino adicional.

O estudo de Lazar (1977) é a primeira tentativa de investigar a transferência de funções entre membros de classes de estímulos. No experimento, um comportamento de ordenação era ensinado a adultos normais. Inicialmente, os participantes deveriam apontar primeiro para um estímulo do par e depois para outro. Em seguida eram submetidos a testes que deveriam avaliar a formação de duas classes ordinais, a dos primeiros e a dos segundos. Posteriormente, novos estímulos eram adicionados às classes existentes através de relações condicionais estabelecidas entre os novos estímulos e os estímulos membros das classes dos primeiros e dos segundos. Pretendia-se verificar o comportamento de ordenação envolvendo esses novos pares de estímulos, demonstrando um desempenho de ordenação com as respectivas classes dos primeiros e dos segundos, sem treino adicional. Os resultados sugeriram que as funções ordinais dos membros das classes dos primeiros e dos segundos transferiram-se para os novos estímulos que tornaram-se relacionados condicionalmente através do matching to sample.

Sigurdardottir e cols. (1990) consideraram que, no estabelecimento de classes com dois estímulos membros, a dos "primeiros" e a classe dos "segundos" (Lazar, 1977), poderia ocorrer algum tipo de restrição relacionada com o controle da seqüência por duas posições de estímulo (Carrigam \& Sidman, 1992). No experimento de Lazar (1977), logo que o sujeito tivesse indicado qual estímulo era o "primeiro" e o estímulo remanescente necessariamente seria o "segundo", desta maneira os participantes não precisariam aprender duas classes ordinais; então o estímulo "segundo" deveria ser tratado como uma classe simples, pois não seria mais relevante assim que uma resposta para o primeiro membro de cada par ocorresse. Estudos posteriores adotaram um número maior de estímulos no treino de seqüenciação.

Diversas táticas para a análise de relações de estímulos dentro de seqüências. Esses procedimentos contam com um tipo de treino de ordenação de estímulos ou seqüenciação e testes para desempenhos seqüenciais produtivos. Um dos paradigmas prevê o estabelecimento de seqüências através do procedimento de encadeamento padrão. Inicialmente, uma seqüência de dois termos é treinada (A1 =>A2), então os demais estímulos são adicionados sucessivamente $(\mathrm{A} 1=>\mathrm{A} 2=>\mathrm{A} 3$, $\mathrm{A} 1=>\mathrm{A} 2=>\mathrm{A} 3=>\mathrm{A} 4, \mathrm{~A} 1=>\mathrm{A} 2=>\mathrm{A} 3=>\mathrm{A} 4=>\mathrm{A} 5)$. Em segui$\mathrm{da}$, testes avaliam a produção das seqüências ensinadas diretamente e seqüências que não foram diretamente ensinadas, mas que já estão incluídas nas seqüências ensinadas. No total são dez pares de estímulos, quatro adjacentes e não seis adjacentes que devem ser testados. Os resultados sugerem que na seqüência ensinada diretamente os estímulos compartilham a propriedade conectividade; isto confronta fortemente a interpretação de que o treino teria estabelecido uma cadeia onde cada estímulo atuou com função discriminativa simples no controle das respostas subsequentes, principalmente quando são avaliados resultados no qual foram apresentados pares de estímulos não adjacentes, pois numa tentativa onde A2 e A4 foram postos juntos, os estímulos discriminativos, ou possíveis elos da cadeia A1 e A3, estiveram ausentes.

Poder-se-ia também sugerir que o treino estabeleceu discriminações condicionais. $\mathrm{O}$ controle condicional não foi explicitamente requerido no treino, mas o desempenho na produção da seqüência de cinco estímulos poderia estar baseado no controle condicional: "se A1 é o primeiro a ser selecionado, selecionar A2 e não A3, A4 ou A5", e assim por diante. Neste caso, a produção de seqüências de dois estímulos nas testes, estaria baseada no desenvolvimento de relações condicionais assimétricas entre estímulos que ocuparam posições adjacentes no treino. Mas, como a propriedade de assimetria não é uma evidência inequívoca do desenvolvimento de uma relação ordinal e seqüências onde ocorrem a ordenação de termos não adjacentes são sempre 
mais informativas, descarta-se o controle exclusivo pela condicionalidade. Logo, o controle condicional discreto é considerado insuficiente para a avaliação de desempenhos corretos em tentativas onde ocorreram pares não adjacentes e onde as dicas condicionais estiveram ausentes.

Procedimentos usados no estudo de inferência transitiva também são úteis na análise do desempenho seqüencial (cf. Stromer \& Mackay, 1990, 1993). O procedimento com pares de estímulos sobrepostos (overllaping two-stimulus) é efetivo no estabelecimento de uma seqüência de cinco estímulos. No treino são estabelecidas quatro seqüências adjacentes $\mathrm{A} 1=>\mathrm{A} 2, \mathrm{~A} 2=>\mathrm{A} 3, \mathrm{~A} 3=>\mathrm{A} 4, \mathrm{~A} 4=>\mathrm{A} 5$. As seqüências $\mathrm{A} 1=>\mathrm{A} 2, \mathrm{~A} 2=>\mathrm{A} 3$ são treinadas diretamente e depois misturadas. Em seguida, ensina-se as seqüências $A 3=>A 4$ e A4=>A5 isoladamente, e os três tipos de tentativas são misturados. Finalmente, os quatro tipos de tentativas são misturados. $\mathrm{O}$ treino com seqüências mistas é mantido até o alcance do critério de aprendizagem e então as testes avaliam a produção de seqüências não ensinadas diretamente. Caso o participante seja capaz de produzir a sequiência completa com os cinco estímulos, avalia-se que o treino tenha estabelecido relações de ordem envolvendo todos os estímulos da seqüência.

Nessa tática, o estímulo A3 aparece em algumas tentativas com A2 e em outras com A4, sendo selecionado como primeiro nas tentativas $\mathrm{A} 3=>\mathrm{A} 4$ e como segundo estímulo nas tentativas $\mathrm{A} 2=>\mathrm{A} 3$. $\mathrm{O}$ desempenho correto nas tentativas $\mathrm{A} 1=>\mathrm{A} 2$ e $\mathrm{A} 4=>\mathrm{A} 5$, que requer discriminações condicionais envolvendo A2 e A4, pode vir a ser útil no estabelecimento de uma regra para discriminações simples no desempenho $\mathrm{A} 2=>\mathrm{A} 4$, permitindo a avaliação da propriedade transitividade. $\mathrm{O}$ desempenho $\mathrm{A} 2=>\mathrm{A} 4$ confirmaria que a relação treinada $\mathrm{A} 2=>\mathrm{A} 3$ e $\mathrm{A} 3=>\mathrm{A} 4$ é transitiva, pois $\mathrm{A} 2$ e A4 nunca apareceram juntos anteriormente (cf. Stromer \& Mackay, 1990).

Outros tipos de arranjos de procedimentos de ensino podem interferir na formação de classes seqüenciais. Uma vez que uma contingência de treino não tenha exigido substitutabilidade entre estímulos de seqüências diferentes, persiste a probabilidade de que alguns participantes não apresentem o desempenho requerido para a avaliação de classes seqüenciais (Stromer \& Mackay, 1993). Um resultado já encontrado indicou que nas tentativas mistas (por exemplo: A1, A3, B2, B4, B5) alguns sujeitos escolheram os estímulos na ordem $\mathrm{A} 1=>\mathrm{A} 3=>\mathrm{B} 2=>\mathrm{B} 4=>\mathrm{B} 5$. Ora, as respostas estavam consistentes com a ordem presente no treino mas não havia qualquer evidência de substitutabilidade entre estímulos de seqüências independentes.

Sigurdardottir e cols. (1990) verificaram que a formação de classes de estímulos e a transferência de funções com adultos normais, era mais provável de ocorrer na condições em que o treino de sequiências precedia o matching. Neste caso, o treino de sequiências poderia estabelecer os pré-requisitos para desempenhos emergentes no matching. Maydack e cols. (1995), no entanto, verificaram que pelo menos entre crianças pequenas (por volta dos 4 anos), as propriedades ordinais adquiridas pelos estímulos no treino de seqüência estabeleceria a base para a formação de classes de estímulos, favorecendo o desempenho emergente no matching, tanto quanto a condição inversa de treino e teste, primeiro o treino com matching to sample e posterior avaliação do desempenho de ordenação. Outros resultados já foram apresentados com indivíduos com déficit comportamental, indicando que o uso do matching to sample, além do estabelecimento de desempenhos seqüenciais como tática de treino pode ser efetivo na formação de classes de estímulos (Stromer, Mackay, Cohen \& Stoddard, 1993; Maydak \& cols. 1995).

No presente estudo, buscou-se avaliar se classes seqüenciais também seriam produzidas após um treino com pareamento consistente modelo-comparação positivo, sem conseqüências diferenciais imediatas. Este procedimento pode permitir inferir o controle por relações de ordem na produção de sequiências novas a partir dos resultados dos testes de assimetria, transitividade e conectividade, avaliando a formação de classes seqüenciais através da múltipla substituição de estímulos.

\section{Método}

\section{Participantes}

Cinco universitários, pertencentes a diversos cursos de graduação, sendo 2 do sexo masculino e 3 do sexo feminino, com faixa etária variando entre 18 a 23 anos. A seleção dos participantes foi feita por contato pessoal. Todos receberam lanches após cada sessão experimental e R\$ 10,00 no final do estudo, independente do seu desempenho.

\section{Ambiente experimental e aparatos}

As sessões experimentais foram realizadas em uma sala do Laboratório de Psicologia Experimental da Universidade Federal do Pará, medindo $6 \mathrm{~m}^{2}$. O participante sentou-se frente a um microcomputador IBM PENTIUM de $133 \mathrm{MHz}$ com tela sensível ao toque com monitor SVGA de 14", policromático. O experimentador posicionou-se ao lado do participante monitorando a sessão. O programa foi elaborado em linguagem VISUAL BASIC 5.0, desenvolvido especialmente para esta pesquisa, a fim de controlar a apresentação de estímulos, números de tentativas, posição de cada estímulo, intervalos entre tentativas (IETs) e registrar as respostas corretas e incorretas.

\section{Estímulos}

Foram usados quatro diferentes conjuntos de estímulos, todos identificados por código alfanumérico (por exemplo, A1, B2 ou C3). No pré-treino e no treino intermediário, foram usadas figuras usuais. No treino de relações condicionais $A B, A C$ e $A D$, foram usados dígitos conhecidos como estímulos modelo e formas geométricas abstratas como estímulos de comparação. Nos testes de seqüenciação, foram usadas apenas formas geométricas abstratas, mencionadas anteriormente. A Figura 1 apresenta os quatro conjuntos de estímulos. No sentido vertical da esquerda para a direita, estão dispostos respectivamente os estímulos dos conjuntos A, B, C e D. No sentido horizontal de cima para baixo, vêse os estímulos que deverão compor as classes ordinais dos "primeiros", "segundos", " terceiros", "quartos" e "quintos" respectivamente. 


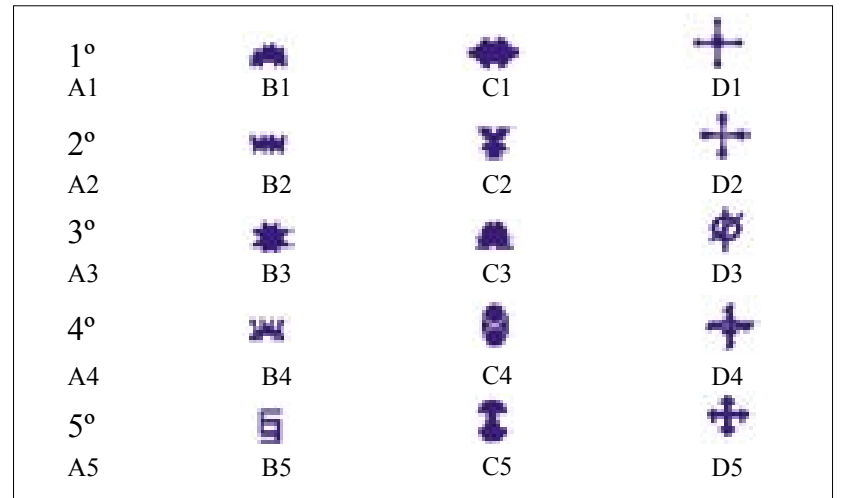

Figura 1. Conjunto de estímulos usados no estudo

\section{Procedimento}

Foi usada apenas uma topografia de resposta de escolha aos estímulos - toque na tela sensível -nas tentativas de treino e testes das relações condicionais e tentativas de testes de produção de seqüências de dois e cinco estímulos. Cada sessão experimental poderia envolver uma seqüência de blocos de tentativas de discriminações condicionais. Cada bloco de treino consistia de tentativas apresentadas aleatoriamente, de modo que a posição dos estímulos de comparação correto e incorretos variasse de tentativa para tentativa.

As tentativas de discriminação condicional iniciavam com a apresentação de um estímulo modelo na "janela" central. Um toque no modelo produzia a apresentação três estímulos de comparação em três das quatro "janelas" laterais, permanecendo o estímulo modelo na tela. A escolha de um estímulo de comparação produzia o término da tentativa, com o desaparecimento dos estímulos e reapresentação da próxima tentativa após um breve intervalo de 1s (IET). Não foram programadas conseqüências diferenciais para respostas corretas ou incorretas em cada tentativa mas o arranjo de estímulos era alterado após um conjunto de quatro tentativas corretas, sendo que o estímulo correto sempre estava presente. No caso de erro, o mesmo conjunto era reapresentado até 10 vezes. Após o treino de relações condicionais, as sessões experimentais envolviam tentativas de seqüenciação de estímulos. Cada bloco consistia de tentativas de testes cuja posição dos estímulos na tela era randomizada, de modo que a posição dos estímulos que deveriam ser ordenados variava de tentativa para tentativa. Uma sessão experimental tinha a duração de 30 minutos, no máximo. Cada participante precisou comparecer no mínimo a quatro sessões para completar sua participação. Mais de uma sessão poderia ser realizada no mesmo dia, de acordo com a disponibilidade do participante.

\section{Seqüência das condições experimentais}

Todos os participantes foram expostos a mesma sequiência de treino e testes. O critério de aquisição de cada discriminação condicional era de $97 \%$ de acertos em um bloco contendo apenas tentativas dessa relação e $100 \%$ de acertos em um bloco misto contendo as relações já ensinadas. Se o critério de acerto não fosse atingido após três apresentações consecutivas do primeiro bloco de treino (A1B1), o participante era exposto ao bloco imediatamente seguinte (por exemplo, A2B2). Caso o participante continuasse respondendo incorretamente em todos os blocos de treino, não seria exposto ao bloco misto, sendo desligado do experimento. A seqüência de fases de treino e testes, com as respectivas relações ensinadas e testadas, é apresentada no Quadro 1. Inicialmente, os participantes foram submetidos ao treino e teste das discriminações condicionais $\mathrm{AB}$ e AC (Fase1). Seguia-se ao treino e teste de uma seqüenciação com cinco estímulos, seqüenciação com pares de estímulos adjacentes e não adjacentes, finalmente, eram realizados os testes de múltipla substituição de estímulos. Em seguida foi realizado o treino e os testes relativos às discriminação condicional $A D$ e finalmente aos testes de múltipla substituição com os estímulos das seqüências $\mathrm{B}, \mathrm{C}$ e D.

Cada participante foi submetido a um pré-treino para familiarização com o equipamento. Um bloco com 12 tentativas nas quais os desenhos de um casa, uma bola e um carro, preenchidos em azul escuro era apresentada e a resposta correta era o desenho casa. A instrução inicial foi fornecida pelo experimentador que solicitou ao participante "Pressione levemente aqui" apontando a janela central; em seguida, "Agora pressione levemente outra janela" e finalmente: "Quando você fizer isto de maneira correta, o computador informará a você" e "Tente acertar o máximo que puder". Um estímulo modelo era apresentado numa janela central, um toque na janela do centro produzia o aparecimento de três estímulos de comparação distribuídas nas janelas laterais. A distribuição das posições dos estímulos era randomicamente programada pelo computador. Uma resposta correta era seguida do aparecimento de uma figura e de uma mensagem na tela: "Muito bem, você acertou" e de uma breve intervalo entre tentativa de 1s (IET). Uma resposta incorreta era seguida pelo IET e nova apresentação da tentativa.

Quadro 1. Seqüência de fases de treino e de testes

\begin{tabular}{ll}
\hline \multicolumn{1}{c}{ Sequiência de Fases } & \multicolumn{1}{c}{ Relações } \\
\hline Treino de discriminações condicionais & $\mathrm{AB} / \mathrm{AC} / \mathrm{AD}$ \\
\hline Teste de simetria & $\mathrm{BA} / \mathrm{CA} / \mathrm{DA}$ \\
\hline Teste de seqüenciação com cinco estímulos & $\mathrm{B} 1=>\mathrm{B} 2=>\mathrm{B} 3=>\mathrm{B} 4=>\mathrm{B} 5 / \mathrm{C} 1=>\mathrm{C} 2=>\mathrm{C} 3=>\mathrm{C} 4=>\mathrm{C} 5 \mathrm{D} 1=>\mathrm{D} 2=>\mathrm{D} 3=>\mathrm{D} 4=>\mathrm{D} 5$ \\
\hline $\begin{array}{l}\text { Teste de seqüenciação com pares de estímulos } \\
\text { adjacentes e não adjacentes para cada conjunto }\end{array}$ & $\mathrm{B} 1=>\mathrm{B} 2 ; \mathrm{B} 1=>\mathrm{B} 3 ; \mathrm{B} 1=>\mathrm{B} 4 ; \mathrm{B} 1=>\mathrm{B} 5 ; \mathrm{B} 2=>\mathrm{B} 3 ; \mathrm{B} 2=>\mathrm{B} 4 ; \mathrm{B} 2=>\mathrm{B} 5 ; \mathrm{B} 3=>\mathrm{B} 4 ; \mathrm{B} 3=>\mathrm{B} 5 ; \mathrm{B} 4=>\mathrm{B} 5$. \\
de estímulos & $\mathrm{D} 1=>\mathrm{D} 2 ; \mathrm{D} 1=>\mathrm{D} 3 ; \mathrm{D} 1=>\mathrm{D} 4 ; \mathrm{D} 1=>\mathrm{C} 5 ; \mathrm{C} 2=>\mathrm{C} 3 ; \mathrm{C} 2=>\mathrm{C} 4 ; \mathrm{C} 2=>\mathrm{C} 5 ; \mathrm{C} 3=>\mathrm{C} 4 ; \mathrm{C} 3=>\mathrm{C} 5 ; \mathrm{C} 4=>\mathrm{C} 5$. \\
\hline Testes de múltipla substituição de estímulos & $\mathrm{C} 1=>\mathrm{B} 2=>\mathrm{C} 3=>\mathrm{B} 4=>\mathrm{B} 5 / \mathrm{B} 1=>\mathrm{D} 1=>\mathrm{D} 4 ; \mathrm{D} 3=>\mathrm{D} 5 ; \mathrm{D} 4=>\mathrm{D} 5$ \\
\hline & $\mathrm{D} 1=>\mathrm{B} 2=>\mathrm{D} 3=>\mathrm{D} 4=>\mathrm{B} 5 / \mathrm{C} 1=>\mathrm{D} 2=>\mathrm{C} 3=>\mathrm{B} 4=>\mathrm{D} 5$ \\
\hline
\end{tabular}




\section{Treino das Discriminações Condicionais}

Todas as relações foram ensinadas através de um arbitrary matching to sample baseado no treino com pareamento consistente modelo-comparação positiva sem conseqüências diferenciais imediatas. Uma relação condicional era ensinada num bloco de 80 tentativas simples (A1B1; A2B2; A3B3; A4B4; A5B5) seguidas de testes para relações simétricas (B1A1; B2A2; B2A3; B4A4; B5A5) e 20 tentativas de treino com tentativas mistas alternadas (baseline) seguidas por uma testes de simetria com 20 tentativas. O procedimento de pareamento consistente, sem consequiências diferenciais em cada tentativa consistiu na apresentação de tentativas aleatórias onde a tarefa do participante era tocar o estímulo consistente (Sc), o estímulo que sempre esteve presente com o modelo, ao longo de 16 tentativas. Em cada tentativa, somente dois dos quatro estímulos de comparação incorretos selecionados aleatoriamente, variando a cada 4 e 8 tentativas, quando ocorria a substituição. O critério de mudança dos estímulos inconsistentes $(\mathrm{Si})$ era 4 respostas corretas seguidas. A seqüência de arranjos de Sc e Si foi aplicada a todos os arranjos de tentativas na Fase1 de forma a garantir as características do pareamento consistente. Por exemplo, Se A1 era modelo e B1 era a comparação positiva, os estímulos inconsistentes variavam a cada quatro tentativas corretas, a partir dos pares B2B3; B3B5; B4B5 e B2B4, respectivamente. O critério de acerto foi $97 \%$ de respostas corretas. Uma resposta incorreta era seguida da reapresentação da tentativa até 10 vezes seguidas.

Após ter alcançado o critério de aquisição na discriminação condicional ensinada, testes das relações simétricas eram apresentadas ao participante imediatamente. Esta antecipação dos testes busca garantir maior efetividade no estabelecimento da discriminação condicional através do treino com pareamento consistente com o modelo por meio da redução do atraso no contato do participante com as contingências de testes (cf. Damin, Assis \& Baptista, 1998).

Um bloco de 20 tentativas de treino misturava os diversos tipos de tentativas. As tentativas eram apresentadas em ordem crescente (A1B1; A2B2; A3B3; A4B4; A5B5). Em seguida, o participante era exposto a um bloco de 20 tentativas de testes de simetria. O Quadro 2 mostra a sequiência de fases de ensino das discriminações condicionais, indicando a ordem em que cada discriminação foi ensinada, o tipo e o número de tentativas por bloco.

O experimentador forneceu aos participantes informações mínimas no início do bloco: "Outras figuras aparecerão na tela e você deverá continuar respondendo", "Quando você fizer isto de maneira correta o computador vai registrar" e "Agora, o computador não informará se você está acertando ou não".

\section{Testes de Seqüenciação de Estímulos}

A tarefa do participante era ordenar os estímulos na ordem em que apareceram no treino. A tela do monitor aparecia dividida em duas áreas. A parte superior era denominada "área de construção seqüencial" e a parte inferior era denominada "área de escolha" (Stromer, Mackay, Cohen \& Soddard, 1993). A instrução "Organize as figuras" era
Quadro 2. Treino de discriminações condicionais intercaladas com testes antecipados, treino misto (baseline) e testes, tipo e número de tentativas.

\begin{tabular}{|c|c|c|c|}
\hline \multirow{2}{*}{ Relaçòes } & \multicolumn{2}{|c|}{ Tipo de Tentativa } & \multirow{2}{*}{$\begin{array}{l}\text { Número de } \\
\text { Tentativas }\end{array}$} \\
\hline & Treino & Testes antecipados & \\
\hline \multirow{12}{*}{$\mathrm{AB} / \mathrm{BA}$} & $\mathrm{A} 1 \mathrm{~B} 1$ & & 16 \\
\hline & & B1A1 & 4 \\
\hline & $\mathrm{A} 2 \mathrm{~B} 2$ & & 16 \\
\hline & & $\mathrm{B} 2 \mathrm{~A} 2$ & 4 \\
\hline & $\mathrm{A} 2 \mathrm{~B} 3$ & & 16 \\
\hline & & B3A3 & 4 \\
\hline & A4B4 & & 16 \\
\hline & & B4A4 & 4 \\
\hline & A5B5 & & 16 \\
\hline & & B5A5 & 4 \\
\hline & Misto AB & & 20 \\
\hline & Simetria BA & & 20 \\
\hline \multirow[t]{12}{*}{$\mathrm{AC} / \mathrm{CA}$} & $\mathrm{A} 1 \mathrm{C} 1$ & & 16 \\
\hline & & C1A1 & 4 \\
\hline & $\mathrm{A} 2 \mathrm{C} 2$ & & 16 \\
\hline & & $\mathrm{C} 2 \mathrm{~A} 2$ & 4 \\
\hline & $\mathrm{A} 2 \mathrm{C} 3$ & & 16 \\
\hline & & $\mathrm{C} 3 \mathrm{~A} 3$ & 4 \\
\hline & $\mathrm{A} 4 \mathrm{C} 4$ & & 16 \\
\hline & & $\mathrm{C} 4 \mathrm{~A} 4$ & 4 \\
\hline & A5C5 & & 16 \\
\hline & & C5A5 & 4 \\
\hline & Misto AC & & 20 \\
\hline & Simetria CA & & 20 \\
\hline \multirow[t]{12}{*}{$\mathrm{AD} / \mathrm{DA}$} & A1D1 & & 16 \\
\hline & & D1A1 & 4 \\
\hline & $\mathrm{A} 2 \mathrm{D} 2$ & & 16 \\
\hline & & $\mathrm{D} 2 \mathrm{~A} 2$ & 4 \\
\hline & A2D3 & & 16 \\
\hline & & D3A3 & 4 \\
\hline & A4D4 & & 16 \\
\hline & & D4A4 & 4 \\
\hline & A5D5 & & 16 \\
\hline & & D5A5 & 4 \\
\hline & Misto AD & & 20 \\
\hline & Simetria DA & & 20 \\
\hline
\end{tabular}

exibida na parte superior da área de construção seqüencial e logo abaixo estava a área reservada para a construção da seqüência. Na parte inferior da tela, estava a área de escolha onde os estímulos eram apresentados randomicamente dentro de células de $2,5 \times 2,5 \mathrm{~cm}$. Uma tentativa iniciava com a apresentação simultânea de todos os estímulos na área de escolha. Um toque em um estímulo produzia seu deslocamento para a área de construção seqüencial. A tentativa era encerrada quando todos os estímulos estivessem organizados na área de construção e a área de escolha estivesse vazia. Duas tentativas corretas seguidas encerraram o teste. Uma tentativa incorreta era seguida por uma nova apresentação da tentativa até o limite de duas tentativas incorretas. Estes critérios foram usados nas testes de seqüenciação com cinco estímulos, testes de seqüenciação com pares de estímulos adjacentes e não adjacentes e testes de múltipla substituição de estímulos. 


\section{Testes de Seqüenciação com cinco estímulos}

A tarefa do participante era ordenar uma sequiência de cinco estímulos, na ordem em que apareceram no treino. $\mathrm{Na}$ presença dos cinco estímulos do conjunto $\mathrm{B}$, o participante deveria tocá-los numa ordem temporal, não importando a posição dos estímulos na área de escolha, de maneira a formar a seqüência $B 1=>B 2=>B 3=>B 4=>B 5$. Na presença dos estímulos do conjunto $\mathrm{C}$, deveria tocar $\mathrm{C} 1=>\mathrm{C} 2=>\mathrm{C} 3=>\mathrm{C} 4=>\mathrm{C} 5$ respectivamente e na presença dos estímulos do conjunto $\mathrm{D}$, deveria tocar $\mathrm{D} 1=>\mathrm{D} 2=>\mathrm{D} 3=>\mathrm{D} 4=>\mathrm{D} 5$ respectivamente. Cada tentativa iniciava com cinco estímulos dispostos na área de escolha e encerrava quando todos estivessem dispostos na área de construção. $\mathrm{O}$ símbolo => foi usado para indicar que um dado estímulo "vem antes" nas respostas de ordenação.

\section{Testes de Seqüenciação com Pares de Estímulos Adjacentes e Não Adjacentes}

A tarefa do participante era ordenar uma sequiência de dois estímulos, na ordem em que apareceram no treino. Foram apresentados 10 pares de estímulos, 4 pares de estímulos que ocuparam posições adjacentes na seqüência de cinco estímulos produzida na testes de seqüienciação com cinco estímulos (ex: B1=>B2, B2=>B3, B3=>B4 e B4=>B5) e 6 pares de estímulos que ocuparam posições não adjacentes na seqüência produzida anteriormente $(\mathrm{ex}$ : $\mathrm{B} 1=>\mathrm{B} 3, \mathrm{~B} 1=>\mathrm{B} 4$, $\mathrm{B} 1=>\mathrm{B} 5, \mathrm{~B} 2=>\mathrm{B} 4, \mathrm{~B} 2=>\mathrm{B} 5, \mathrm{~B} 3=>\mathrm{B} 5)$. Cada tentativa iniciava com dois estímulos dispostos na área de escolha e encerrava quando todos estivessem dispostos na área de construção.

\section{Testes de Múltipla Substituição de Estímulos}

A tarefa do participante era organizar uma seqüência de cinco estímulos utilizando os estímulos disponíveis na área de escolha. Este tipo de testes foi apresentado pela primeira vez após a apresentação das testes de seqüenciação com cinco estímulos do conjunto $\mathrm{C}$, onde estímulos da seqüência $\mathrm{B}$ e $\mathrm{C}$ eram apresentados randomicamente na área de escolha. Após a exposição às testes relativas ao conjunto $\mathrm{D}$, a testes de múltipla substituição de estímulos consistia na apresentação randomizada de estímulos provenientes das seqüências $\mathrm{B}, \mathrm{C}$ e D formadas anteriormente. Uma tentativa iniciava com a disposição de cinco estímulos na área de escolha e encerrava quando estes estímulos estivessem dispostos na área de construção

\section{Resultados}

\section{Análise do desempenho no treino de discriminação condicional}

Dos cinco participantes, três atingiram $100 \%$ de respostas corretas em cada bloco de discriminação condicional ensinada na de linha de base. Este nível de desempenho foi mantido durante e após as testes para esses participantes. A Tabela 1 apresenta o número de blocos que cada participante precisou até atingir o critério de acertos estabelecido para cada discriminação condicional e na linha de base. Dois outros participantes não alcançaram o critério de acerto e foram dispensados.

Os participantes precisaram de maior número de blocos para atingir o critério de acertos nas primeiras discriminações condicionais ensinada $(A B)$ do que nas demais discriminações do treino de aquisição. Apenas um participante (ATI) precisou de maior número de blocos para atingir o critério de acertos na segunda discriminação condicional ensinada $(A C)$. Os outros dois participantes precisaram de duas exposições em apenas um bloco da discriminação condicional $A D$ cada um. Nos blocos mistos não houve necessidade de reexposição para todos os que alcançaram o critério (ver Tabela 1).

\section{Análise do desempenho nas testes de seqüenciação com 5 estímulos}

Dos três participantes que foram expostos aos testes de seqüenciação com cinco estímulos, dois formaram todas as

Tabela 1. Número de respostas corretas por participante em cada bloco de treino das relações $\mathrm{AB}, \mathrm{AC}$ e $\mathrm{AD}$.

\begin{tabular}{|c|c|c|c|c|}
\hline & & \multicolumn{3}{|c|}{ Respostas Corretas por participante } \\
\hline & & MOI & LUC & ATI \\
\hline Treino & Teste & & & \\
\hline \multirow[t]{2}{*}{ A1B1 } & & $15 / 16$ & $15 / 16$ & $16 / 16$ \\
\hline & Testes B1A1 & $4 / 4$ & $4 / 4$ & $4 / 4$ \\
\hline \multirow[t]{2}{*}{$\mathrm{A} 2 \mathrm{~B} 2$} & & $16 / 16$ & 1616 & $16 / 16$ \\
\hline & testes B2A2 & $4 / 4$ & $4 / 4$ & $4 / 4$ \\
\hline \multirow[t]{2}{*}{ A3B3 } & & $16 / 16$ & $16 / 16$ & $16 / 16$ \\
\hline & testes B3A3 & $4 / 4$ & $4 / 4$ & $4 / 4$ \\
\hline \multirow[t]{2}{*}{ A4B4 } & & $16 / 16$ & $15 / 16$ & $16 / 16$ \\
\hline & testes B4A4 & $4 / 4$ & $4 / 4$ & $4 / 4$ \\
\hline \multirow[t]{2}{*}{ A5B5 } & & $16 / 16$ & $16 / 16$ & $16 / 16$ \\
\hline & testes B5A5 & $4 / 4$ & $4 / 4$ & $4 / 4$ \\
\hline \multirow[t]{2}{*}{$\mathrm{AB}$ misto } & & $20 / 20$ & $20 / 20$ & $20 / 20$ \\
\hline & Simetria BA & $20 / 20$ & $20 / 20$ & $20 / 20$ \\
\hline \multirow[t]{2}{*}{$\mathrm{A} 1 \mathrm{C} 1$} & & $15 / 16$ & $16 / 16$ & $16 / 16$ \\
\hline & testes $\mathrm{C} 1 \mathrm{~A} 1$ & $4 / 4$ & $4 / 4$ & $4 / 4$ \\
\hline \multirow[t]{2}{*}{$\mathrm{A} 2 \mathrm{C} 2$} & & $16 / 16$ & $16 / 20$ & $16 / 16$ \\
\hline & testes $\mathrm{C} 2 \mathrm{~A} 2$ & $4 / 4$ & $4 / 4$ & $4 / 4$ \\
\hline \multirow[t]{2}{*}{$\mathrm{A} 3 \mathrm{C} 3$} & & $15 / 16$ & $15 / 16$ & $16 / 16$ \\
\hline & testes $\mathrm{C} 3 \mathrm{~A} 3$ & $4 / 4$ & $4 / 4$ & $4 / 4$ \\
\hline \multirow[t]{2}{*}{$\mathrm{A} 4 \mathrm{C} 4$} & & $15 / 16$ & $14 / 24$ & $16 / 16$ \\
\hline & testes $\mathrm{C} 4 \mathrm{~A} 4$ & $4 / 4$ & $4 / 4$ & $4 / 4$ \\
\hline \multirow[t]{2}{*}{ A5C5 } & & $15 / 16$ & $15 / 16$ & $16 / 16$ \\
\hline & testes C5A5 & $4 / 4$ & $4 / 4$ & $4 / 4$ \\
\hline \multirow[t]{2}{*}{ AC misto } & & $20 / 20$ & $20 / 20$ & $20 / 20$ \\
\hline & Simetria CA & $20 / 20$ & $20 / 20$ & $20 / 20$ \\
\hline \multirow[t]{2}{*}{ A1D1 } & & $16 / 16$ & $15 / 16$ & $16 / 16$ \\
\hline & testes D1A1 & $4 / 4$ & $4 / 4$ & $4 / 4$ \\
\hline \multirow[t]{2}{*}{ A2D2 } & & $16 / 16$ & $16 / 16$ & $16 / 16$ \\
\hline & testes D2A2 & $4 / 4$ & $4 / 4$ & $4 / 4$ \\
\hline \multirow[t]{2}{*}{ A3D3 } & & $16 / 16$ & $16 / 20$ & $16 / 16$ \\
\hline & testes D3A3 & $4 / 4$ & $4 / 4$ & $4 / 4$ \\
\hline \multirow[t]{2}{*}{ A4D4 } & & $15 / 16$ & $15 / 16$ & $16 / 16$ \\
\hline & testes D4A4 & $4 / 4$ & $4 / 4$ & $4 / 4$ \\
\hline \multirow[t]{2}{*}{ A5D5 } & & $15 / 16$ & $16 / 16$ & $16 / 16$ \\
\hline & testes D5A5 & $4 / 4$ & $4 / 4$ & $4 / 4$ \\
\hline \multirow[t]{2}{*}{ AD misto } & & $20 / 20$ & $20 / 20$ & $20 / 20$ \\
\hline & Simetria DA & $20 / 20$ & $20 / 20$ & $20 / 20$ \\
\hline
\end{tabular}


sequiências com $100 \%$ de acerto. O participante LUC apresentou erro na formação da primeira sequiência solicitada e acertou as demais. A Tabela 2 apresenta o número de acertos nas testes de seqüenciação de cinco estímulos.

Tabela 2. Resultados nas testes de seqüenciação com cinco estímulos

\begin{tabular}{cccc}
\hline \multicolumn{4}{c}{ Número de acertos de cada } \\
participante por número total de tentativas \\
\hline \multicolumn{1}{c}{ Seqüência } & MOI & LUC & ATI \\
\hline $\mathrm{B} 1=>\mathrm{B} 2=>\mathrm{B} 3=>\mathrm{B} 4=>\mathrm{B} 5$ & $2 / 2$ & $1 / 2$ & $2 / 2$ \\
$\mathrm{C} 1=>\mathrm{C} 2=>\mathrm{C} 3=>\mathrm{C} 4=>\mathrm{C} 5$ & $2 / 2$ & $2 / 2$ & $2 / 2$ \\
$\mathrm{D} 1=>\mathrm{D} 2=>\mathrm{D} 3=>\mathrm{D} 4=>\mathrm{D} 5$ & $2 / 2$ & $2 / 2$ & $2 / 2$ \\
\hline
\end{tabular}

\section{Análise dos desempenhos nas testes de seqüenciação com pares de estímulos adjacentes e não adjacentes}

Os participantes MOI e ATI acertaram 100\% das tentativas com pares de estímulos adjacentes e não adjacentes baseadas nas três seqüências de cinco estímulos produzidas anteriormente (ver Quadro 1). O participante LUC não obteve acertos em uma tentativa com pares de estímulos adjacentes e não adjacentes baseada na seqüência com cinco estímulos provenientes do conjunto $\mathrm{B}$, acertou apenas $33 \%$ em oito tentativas; acertou $50 \%$ em uma tentativa e não obteve acertos em outra. Nos demais testes baseados nas sequiências C e D, o participante acertou $100 \%$ em todas as tentativas.

\section{Análise do desempenho nas testes de múltipla substituição de estímulos}

Na literatura, o critério de $90 \%$ de respostas corretas em qualquer bloco de tentativas de testes é adotado como indicador de transferência de funções discriminativas e formação de classes de equivalência. Desse modo, adotamos o mesmo critério para sugerir a formação de classes sequiências neste estudo. A emergência imediata de desempenho seqüencial produtivo foi considerada quando o participante atingiu este critério na primeira apresentação do bloco de testes e emergência gradual quando este critério foi alcançado nas apresentações subsequentes.

Dos três participantes apenas LUC não atingiu $100 \%$ de acerto na primeira apresentação nas testes de múltipla substituição de estímulos. Os dois outros participantes mostraram emergência imediata de desempenho produtivo indicando o estabelecimento de relações de ordem entre membros de sequiências diferentes na primeira exposição aos testes. A Tabela 3 apresenta o número de acertos na testes de múltipla substituição de estímulos.

Tabela 3. Resultados dos participantes MOI, LUC e ATI nas testes de múltipla substituição de estímulos

\begin{tabular}{cccc}
\hline \multicolumn{4}{c}{ Número de acertos de cada } \\
peqticipante por número total de tentativas \\
\hline Sequiência & MOI & LUC & ATI \\
\hline $\mathrm{B} 1=>\mathrm{C} 2=>\mathrm{C} 3=>\mathrm{B} 4=>\mathrm{C} 5$ & $2 / 2$ & $1 / 2$ & $2 / 2$ \\
$\mathrm{C} 1=>\mathrm{B} 2=>\mathrm{C} 3=>\mathrm{B} 4=>\mathrm{B} 5$ & $2 / 2$ & $1 / 2$ & $2 / 2$ \\
$\mathrm{D} 1=>\mathrm{B} 2=>\mathrm{D} 3=>\mathrm{D} 4=>\mathrm{B} 5$ & $2 / 2$ & $2 / 2$ & $2 / 2$ \\
$\mathrm{C} 1=>\mathrm{D} 2=>\mathrm{C} 3=>\mathrm{B} 4=>\mathrm{D} 5$ & $2 / 2$ & $2 / 2$ & $2 / 2$ \\
\hline
\end{tabular}

Nos resultados obtidos com os participantes MOI, LUC ATI, os dados de latência relativa à escolha de cada estímulo nas testes de seqüenciação, indicaram que os participantes responderam mais lentamente aos estímulos "primeiro" que aos demais, e a duração da resposta também reduzia ao logo de cada tentativa nos testes. Esses resultados podem ser avaliados ainda com base nos estudos sobre os efeitos de variáveis estruturais como posição serial ou distância simbólica que avaliam o controle por end-anchored (Holcomb \& cols.,1997). Mas, esta ainda é uma questão a ser melhor esclarecida, uma vez que este treino não envolveu seqüências sobrepostas (overlapping), tendo envolvido um matching to sample para estabelecer o desempenho de ordenação, portanto, os estímulos primeiro e último não foram apresentados juntos no treino antes das testes de seqüenciação.

\section{Discussão}

Cinco participantes foram submetidos a um procedimento de pareamento consistente modelo-Sc, onde foram solicitados a relacionar um conjunto de dígitos ou numerais ordinais (modelos) com três conjuntos diferentes de formas geométricas abstratas (comparações), sem consequiências diferenciais imediatas. Três participantes foram capazes de relacionar condicionalmente os dígitos com as formas e apresentar comportamento de ordenação com base nas relações condicionais aprendidas. Este desempenho emergente sugeriu que houve a formação de quatro classes ordinais com quatro membros cada uma, consistindo de um numeral ordinal e três formas geométricas diferentes. Estes resultados estão de acordo com a literatura sobre a formação e expansão de classes de estímulos ordinais (Lazar, 1977; Sigurdardottir \& cols., 1990).

Os resultados do presente trabalho estendem os observados na literatura com esse procedimento de pareamento consistente modelo-Sc (Williams \& cols., 1995; Baptista \& Assis, 1995; Assis \& cols., 1997; Damin \& cols., 1998; Barros \& cols., 1998; Saunders \& cols., 1999; Assis \& cols., 2000; Álvares \& cols., 2001), sugerindo que o treino com pareamento consistente é uma tática alternativa para produzir classes de estímulos na ausência de consequiências diferenciais em cada tentativa.

A verificação de classes seqüenciais pressupõe que todos os estímulos na classes sejam mutuamente substituíveis no controle de um mesmo desempenho, e que qualquer propriedade controladora adquirida por um membro da classe deva ser compartilhado por todos os outros membros. $\mathrm{O}$ estudo da transferência de controle de estímulos usando um procedimento de matching to sample para a produção de sequiências configura-se como uma tática para avaliar a formação de relações de ordem, pré-requisitos para a formação de classes seqüenciais.

Para que um resultado sobre a emergência de seqüências seja considerado confiável, deve sugerir que o treino estabeleceu relações ordinais entre os estímulos. Neste estudo, os resultados de três participantes indicaram que os cinco estímulos condicionalmente relacionados no treino, formavam um conjunto ordenado que partilhavam as mesmas propriedades de estímulos no contexto de formação de seqüências. As propriedades de estímulos avaliadas nas testes parecem 
indicar um exemplo de emergência imediata, pelo menos para dois dos participantes (MOI e ATI) e gradual para um (LUC), uma vez que estiveram baseadas no desempenho produtivo requerido pelo contexto de formação de sequiências. Assim que foram solicitados a formar sequiências de cinco estímulos, esses participantes organizaram os estímulos apresentados, selecionando-os de forma a indicar o requisito de unidirecionalidade, de acordo com o que estabelece a propriedade de assimetria.

Foi possível inferir a propriedade de transitividade a partir dos resultados nas testes com dois estímulos. Os três participantes, mesmo que de forma gradual (LUC), organizaram os pares de estímulos que ocuparam posições não adjacentes na sequiência de cinco estímulos na ausência de um estímulo discriminativo que poderia ter funcionado como elo. Isto é, o desempenho B2=>B4 foi apresentado, mesmo na ausência de um suposto estímulo discriminativo (B3), indicando desta forma que o desempenho não estaria baseado no encadeamento, e replicando os achados acerca do potencial transitivo que poderia estar baseado na condicionalidade estabelecida no contexto de matching to sample (Green et al, 1993; Stromer \& Mackay, 1993; Sigurdadotir \& cols., 1990 e Lazar, 1977). E mais, especialmente neste estudo, a propriedade transitividade parece estar relacionada com as relações aprendidas no treino de linha de base e que apareciam inseridas da seqüência de cinco estímulos, sem que houvesse ocorrido treino explícito. Neste caso, se B2 $=>B 3$ e B3=>B4, então B2=>B4. Este desempenho não foi requerido pelo treino, mas as relações condicionais das quais a transitividade poderia ser inferida parecem ter sido a base para a produção das seqüências de cinco estímulos novas; desta maneira, este pode ser considerado um desempenho novo, ou produtivo.

Tanto as testes de seqüenciação de cinco estímulos quanto as testes de seqüienciação com pares de estímulos adjacentes e não adjacentes, sugeriram que as propriedades controladoras adquiridas por um membro da classe estava sendo compartilhado por todos os outros membros, favorecendo a inferência da propriedade de conectividade. Portanto, os resultados deste experimento estão baseados nos pressupostos sugeridos por Green e cols. (1993) para a estruturação de testes para as propriedades de relações ordinais, estabelecidas entre estímulos de uma seqüência e entre seqüências independentes.

Os resultados acerca da formação de classes de estímulos indicaram que todos os três participantes apresentaram 100\% de acerto nas testes de múltipla substituição de estímulos, tanto para aquelas que misturaram estímulos dos conjuntos $\mathrm{B}$ e $\mathrm{C}$, quanto aquelas que misturaram estímulos dos conjuntos B, C e D. Os três participantes formaram sequiências de cinco estímulos, substituindo estímulos que ocuparam a mesma posição em sequiências diferentes, não importando a disposição espacial em que os estímulos apareceram na área de escolha. Este desempenho é a base para a inferência de formação de classes seqüenciais.

Recentemente, resultados que envolvem a latência de respostas nas sequiências de linha de base, têm sido considerados na avaliação do tipo de controle envolvido no treino que estabeleceu relações ordinais. Holcomb, Stromer e Mackay, (1997) buscaram verificar a partir deste tipo de análise, os efeitos da posição serial e da distância na produção de seqüências. Para esses autores, tais aspectos parecem sugerir diferenças no controle de estímulos envolvidos nas tentativas que envolvem o estímulo final (end-anchored) e seqüências sobrepostas (overlapping) Os estudos que investigam esses aspectos, estão relacionados com a tentativa de determinar a natureza do controle de estímulos estabelecido por variáveis estruturais de treino específicas, e pode ser uma estratégia similar àquelas que investigam os efeitos da distância nodal no estabelecimento de relações condicionais. (Fields \& Verhave, 1987), pois ambas referem-se a um tipo de variável experimental que pode ter implicações relacionadas com o potencial das características estruturais. Mas outras propostas alternativas vêm sendo sugeridas, como a que pressupõe a avaliação dos resultados diferenciais no estabelecimento de classes de equivalência com base na análise de discriminações simples e sucessivas que podem ser estabelecidas no treino e teste de discriminações condicionais (Saunders \& Green, 1999). Estes podem favorecer novos caminhos para a análise no âmbito do desempenho seqüencial produtivo, que não foi, entretanto o objetivo do presente trabalho.

A principal contribuição desse estudo está relacionada com a efetividade do procedimento de treino com pareamento consistente sem conseqüências diferenciais imediatas, para produzir classes seqüenciais.

\section{Referências}

Adams, B.J., Fields L. \& Verhave T. (1993). Formation of generalized equivalence classes. The Psychological Record, 43, 553-566.

Assis, G.J.A., Baptista, M.Q.G., Damin, E.T.B., \& Álvares, S.M.M. (1997). Consistency training and equivalence relations without differential consequences. Experimental Analysis of Human Behavior Bulletin, 15 (1), 6-7

Álvares, S.M.M., Assis, G.J.A. Sampaio, M.E.C.E. e Esteves, I.P. (2001). Discriminações condicionais sem consequiências diferenciais em crianças: efeitos da história de treino e teste precoce de simetria. Estudos de Psicologia, 6 (1), 11-24

Assis, G.J.A., Baptista, M.Q.G., Kato, O.M., \& Alves, K.R.S. (2000). Relações de equivalência após treino com pareamento consistente de estímulos sob controle contextual. Psicologia: Teoria e Pesquisa, 16 (2), 125-133

Baptista, M.Q.G. \& Assis, G.J.A. (1995). Treino por consistência de estímulos sem consequiências diferenciais. Psicologia: Teoria e Pesquisa, 11, 173-179.

Barros, C.W.L., Baptista, M.Q.G. \& Assis, G.J.A. (1998). Efeitos de história de treino sobre a formação de classes de estímulos equivalentes. Acta Comportamentalia, 6, 111-128.

Carrigan, P.F. \& Sidman, M. (1992).Conditional discrimination and equivalence relations: a theoretical analysis of control by negative stimuli. Journal of the Experimental Analysis of Behavior. 58, 459-504.

Catania, A.C. (1980). Autoclit process and the structure of behavior. Behaviorism, 175-186.

Damin, E.T.B., Assis, G.J.A. \& Baptista; M.Q.G. (1998). Efeitos da distribuição de treino /testes sobre a formação de classes de estímulos equivalentes sem conseqüências diferenciais. Psicologia : Teoria e Pesquisa, 14, 041 - 049.

Fields, L e Verhave, T. (1987). The structure of equivalence 
classes. Journal of the Experimental Analysis of Behavior, 48, 317-332.

Green, G., Sigurdardottir, Z.G. \& Saunders, R.R. (1991). The role of instructions in transfer of ordinal functions thorugh equivalence classes. Journal of the Experimental Analysis of Behavior, 55, 287-304.

Green, G., Stromer, R. \& Mackay, H. (1993). Relational learning in stimulus sequences. The Psychological Record, 43, 599-616

Harrison, R.J. \& Green, G. (1990). Development of conditional and equivalence relational without diferencial consequences. Journal of the Experimental Analysis of Behavior, 54, 225, 237.

Holcomb, W.L., Stromer, R., \& Mackay, H.A. (1997). Transitivity and emergent sequence performance in young children. Journal of Experimental Child Psychology, 65, 96-124.

Lazar, R. (1977). Extending sequence-class membership with matching to sample. Journal of the Experimental Analysis of Behavior, 27, 381-392

Maydak, M., Stromer, R. Mackay, H., \& Stoddard, L. (1995). Stimulus classes in matching to sample and sequence production: the emergence of numeric relations. Research in Developmental Disabilities, 16, 179-204.

Saunders, R.R., \& Green, G. (1999). A discrimination analysis of training-structure effects of stimulus equivalence outcomes. Journal of the Experimental Analysis of Behavior, 72, 117137.

Saunderes, R.R., Drake, K.M., \& Spradlin, J.E. (1999). Equivalence class establishment expansion, and modification in preschool children. Journal of the Experimental Analysis of Behavior, 71, 195-214

Sidman. M. (1986). Functional Analysis of Emergent Verbal Classes. In: Thompson, T. \& Zeiler, M.D.L (Org.). Analysis and Integration of Behavioral Units (p.213-245). N.J.: Lawrence Erlbaum Associates Publishers.
Sidman. M. (1994). Equivalence Relations and Behavior: A Research Story. Boston: Authors Cooperative, Inc. Publishers.

Sidman, M. \& Tailby, W. (1982). Conditional discrimination versus matching-to-sample: An expansion of the testing paradigm. Journal of the Experimental Analysis of Behavior, 37, 5-22.

Sigurdardottir, Z.G.; Green, G. \& Saunders, R.R. (1990). Equivalence classes generated by sequence training. Journal of the Experimental Analysis of Behavior, 53, 47-63.

Skinner, B.F. (1938). The Behavior of Organisms. New York: Appleton-Century-Crofts.

Skinner, B.F. (1953). Science and Human Behavior. New York: The Macmillan Company

Skinner, B.F. (1957). Verbal Behavior. New York: Prentice-Hall, Inc.

Stromer, R. \& Mackay, H.A. (1990). A note on the study of transitive relation in stimulus sequence. Experimental Analysis of Human Behavior Bulletin, 8, 2-5.

Stromer, R. \& Mackay, H.A. (1992). Conditional stimulus control of children's sequence production. Psychological Reports, 70, 903-912.

Stromer, R. \& Mackay, H.A. (1993). Human sequential behavior: Relations among stimuli, class formation, and derived sequences. The Psychological Record, 43, 107-131.

Stromer, R., Mackay, H.A, Cohen, M., \& Stoddard, L.T. (1993). Sequence learning in individuals with behavioral limitations. Journal of Intellectual Disability Research, 37, 243-261.

Williams, D.C., Saunders,. K.J., Saunders, R.R., \& Spradlin, J.E (1995). Unreinforced conditional selection within three choice conditional discriminations. The Psychological Record, 45, 613-627. 